\title{
Integration of a 3D Hydrogel Matrix with a Hollow Core Photonic Crystal Fibre for DNA Probe Immobilisation
}

\author{
Monika S Rutowska ${ }^{1}$, Fatima C Garcia Gunning ${ }^{1}$, Francine Kivlehan ${ }^{2}$, Eric Moore $^{2}$, Des \\ Brennan², Paul Galvin², Andrew D Ellis $^{1}$ \\ ${ }^{1}$ Photonic System Group and Department of Physics \\ ${ }^{2}$ NanoBio Systems Group \\ Tyndall National Institute, University College Cork, Ireland \\ E-mail: monika.rutowska@tyndall.ie
}

\begin{abstract}
In this paper, we demonstrate the integration of a 3D hydrogel matrix within a hollow core Photonic Crystal Fibre (HC-PCF). In addition we also show the fluorescence of Cy5-labelled DNA molecules immobilised within the hydrogel formed in two different types of HC-PCF. The 3D hydrogel matrix is designed to bind with the amino-groups of biomolecules using an appropriate cross-linker, providing higher sensitivity and selectivity than the standard 2D coverage, enabling a greater number of probe molecules to be available per unit area. The HC-PCFs, on the other hand, can be designed to maximise the capture of fluorescence to improve sensitivity and provide longer interaction lengths.This could enable the development of fibre-based point-of-care and remote systems, where the enhanced sensitivity would relax the constraints placed on sources and detectors. In this paper, we will discuss the formation of such polyethylene glycol diacrylate (PEGDA) hydrogels within a HC-PCF, including their optical properties such as light-propagation and auto-fluorescence.
\end{abstract}

KEYWORDS:

Photonic crystal fibres, hollow core, bio sensing, hydrogel, fluorescence, DNA immobilisation, silanisation

\section{Introduction}

Optical fibre sensors have been investigated over many decades and recently sensors using Photonic Crystal Fibres (PCF) and microstructure optical fibres (MOF) have shown great potential for a number of applications. Unlike standard optical fibres, PCFs and certain MOFs have a photonic crystal-like structure surrounding the core, which enables light to be propagated through the core without the assistance of codopants $[1,2]$. Hollow core photonic crystal fibre (HC-PCF) which operate through the photonic bandgap effect (PBG) [3,4,5,6,7] offer the prospect of common confinement of the sample and the optical field. In a PBG fibre only a restricted band of wavelengths is confined in the core by the photonic bandgap effect induced by a microstructure fabricated within the cladding. Samples which are typically confined to the hollow core of the PBG fibre have improved the interaction between light within the guided wavelength band and the sample under test [8].

Recently, bio sensing using glass PCFs [9], glass MOFs [10], and polymer MOFs [11] were verified, and the possibility of antibody immobilisation within the microstructure has been demonstrated $[9,12]$. The use of such fibres enables biosensors with high sensitivity, because the interaction length can be extended to several centimetres if not meters, and fibres can also be designed to maximise fluorescence guidance [13]. They also enable selectivity, with the possibility for multiple antigens to be attached and analysed with a multi-wavelength optical source. HC-PCFs have been used for immobilisation, capture and detection of DNA probe molecules [14]. However, such immobilisation techniques are based on standard techniques for surface coating on a two dimensional (2D) scale. 
Lately, three dimensional (3D) hydrogels have been demonstrated as alterative substrates for microarray applications [15] with higher sensitivity [16,17] than the 2D matrices when grown on glass substrates. Hydrogels (also called aquagels) are mostly liquid, yet behave like solids due to a threedimensional crosslinked network within the lattice structure. They consist of a group of polymer chains that are water-insoluble. Hydrogels contain binding sites for the covalent attachment of biological compounds within their porous structure via the use of an appropriate cross-linking agent. For the immobilisation of probe DNA molecules, they are first modified with an amino group in order to allow for successful attachment to the polymer backbone. The immobilisation occurs simultaneously during the formation of the hydrogel, in a photo-polymerisation process [18].

The advantages of using a hydrogel include the increased capacity for the immobilisation of the biomolecules, due to a greater number of probe molecules to available per unit area. The hydrogel's high affinity for water can provide a homogeneous environment with the surrounding aqueous solution, which allows for the free diffusion or mass transfer of the biomolecules into the hydrogel matrix. This increases the probability of target DNA molecules of binding to and interacting with complementary probes immobilised within the hydrogel matrix. They also offer the possibility of reducing steric hindrence which is a factor contributing to sensitivity and reproducibility limits in the most of 2D systems [16,17].

In this paper we demonstrate the immobilisation and fluorescent detection of a Cy5-labelled DNA probe in a polyethylene glycol diacrylate (PEGDA) hydrogel platform formed within a glass-based HC-PCF. We show, for the first time, the studies of light propagating through the hydrogel structure, indicating the refractive index of the hydrogel within the fibre.

\section{HC-PCF preparation}

\subsection{HC-PCF samples}

Short pieces $(10$ to $30 \mathrm{~cm}$ ) of commercially available HC-PCFs (NKT Photonics A/S) were used, which were originally designed for 1060 and $1550 \mathrm{~nm}$ propagation (HC-PCF 1060 and HC-PCF 1550), with core diameters of approximately 9.8 and $11.0 \mu \mathrm{m}$ respectively (figure 1 ).

(a)

(b)

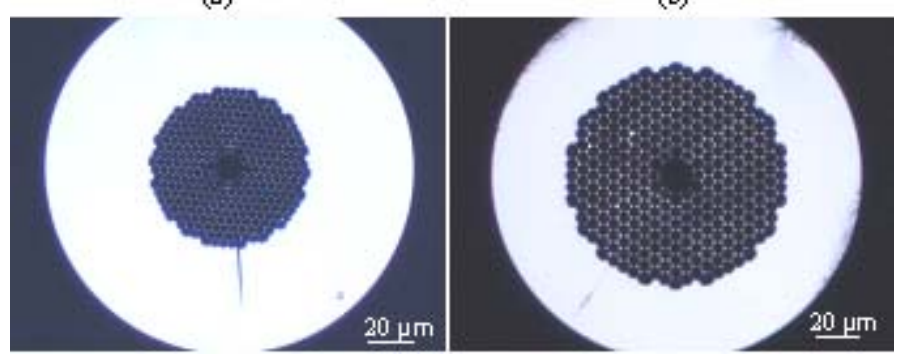

Figure 1: Cross section of fibres (a) HC-PCF 1060 and (b) HC-PCF 1550.

The chemical preparation of the hole surfaces and the photo-initiation of the 3D hydrogel took place within the hollow core and the cladding holes, although selective filling could also have been used [19]. Instead of relying on a lengthy filling process based on capillary action, a syringe pump (KD Scientific 410) was used to assist both the chemical filling and rinsing. Each chemical solution described below was pumped for about 5 minutes, even though the hollow core of the PCF can be filled within seconds and were kept incubated within the microstructure for a certain period of time for each step. This protocol was followed in order to ensure a good surface coverage. All chemicals and reagents used were purchased from Sigma-Aldrich Ltd, unless stated otherwise.

\subsection{Silanisation process and immobilisation of Eosin $Y$}

The holes of the HC-PCFs were chemically treated as per [20]. The holes were pumped with a 3\% (v/v) solution of (3-Aminopropyl) trimethoxysilane (APTMS), which was prepared in 19:1 solution of methanol and deionised water. The filling process was monitored via a microscope (Nikon Eclipse ME600), where one 
of the fibre ends was held vertically and its cross-section observed; and the other end was attached to the syringe pump. This solution of silanising agent was kept in the fibre and incubated for 1 hour at room temperature. Afterwards, the silanising solution was removed by several rinses of methanol and cured in an oven for 2 hours, at $60^{\circ} \mathrm{C}$. A $10 \mathrm{mM} \mathrm{NaH}{ }_{2} \mathrm{PO}_{4}$ solution was prepared in order to make up a solution containing $0.5 \mathrm{mM}$ Eosin $\mathrm{Y}$ and $25 \mathrm{mM}$ N-(3-Dimethylaminopropyl)-N'-ethylcarbodiimide hydrochloride (EDAC) and the resultant solution was pumped into the fibre (figure 2(b)). After 90 minutes, the sample was rinsed with deionised water and dried in an oven at $35^{\circ} \mathrm{C}$ for 15 hours. After this process, a green reflection from the fibre was observed using microscope, indicating successful Eosin Y immobilisation on the silanised surface (figure 2 (c)).

(a)

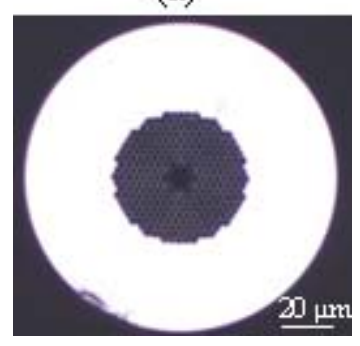

(b)

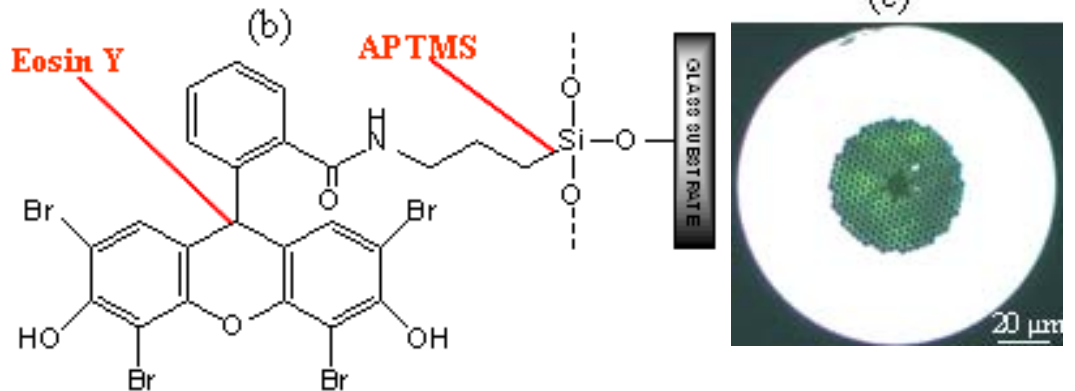

Figure 2: Cross section of HC-PCF 1060 (a) before a silanisation process, (b) the silanised glass substrate with attached Eosin Y, (c) HC-PCF 1060 after a silanisation process.

\subsection{Photochemical initiation and hydrogel growth}

A pre-polymer solution was prepared in phosphate buffer solution (PBS), consisting of 25\% (v/v) poly(ethylene glycol) diacrylate (PEGDA), $225 \mathrm{mM}$ triethanolamine, and $37 \mathrm{mM}$ 1-vinylpyrollidinone. Solutions were stored in the fridge at $\sim 4^{\circ} \mathrm{C}$. The pre-polymer solution was deposited into the HC-PCF fibres which were previously functionalised with silane anchor molecules and Eosin Y photo-initiator, and then irradiated with visible light at a wavelength of $530 \mathrm{~nm}$ for about 6 minutes; and the optical setup for the hydrogel growth is shown in figure 3.

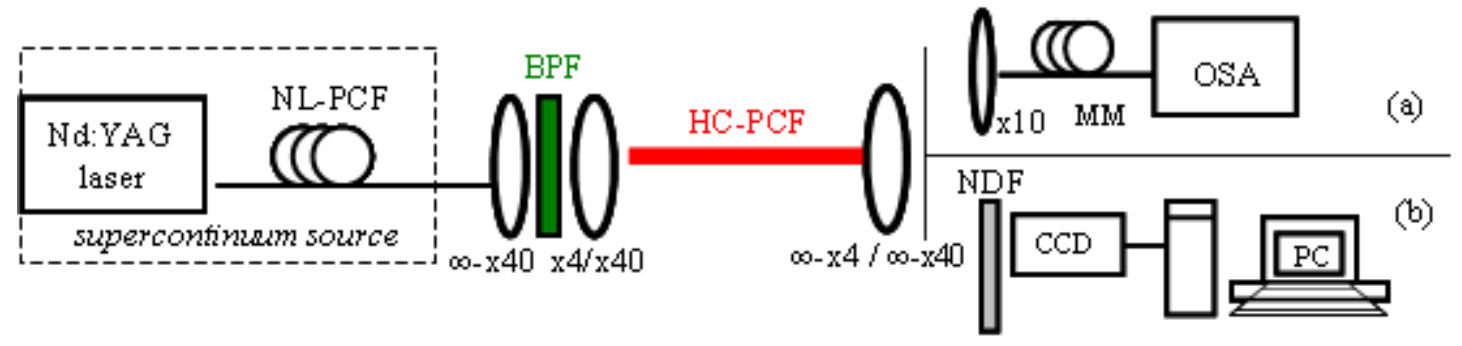

Figure 3: Optical experimental setup used for photochemical initiation and spectral analysis.BPF = bandpass filters, $\mathrm{MM}=$ multimode fibre, OSA = optical spectrum analyser, NDFs - neutral density filters, $\mathrm{CCD}$ = camera, PC=desktop (a) spectrum analysis and (b) near-field images.

A Q-Switched semiconductor pumped Nd:YAG laser (JDS Uniphase), centred at $1064 \mathrm{~nm}(6.85 \mathrm{kHz}$ repetition rate, 0.55 ns pulse width, and a $\sim 10.7 \mu \mathrm{J} /$ pulse) together with a $20 \mathrm{~m}$ highly nonlinear PCF (NLPCF) were used as a supercontinuum source [21]. The light was coupled to the fibre by a combination of collimating and focusing lenses. Photochemical initiation of the polymerization process was achieved by filtering the supercontinuum spectrum using a broad bandpass filter (10 nm FWHM) centred at $530 \mathrm{~nm}$. Typically 5 to 10 minutes was sufficient for hydrogel formation through the entire length of the fibre, with average input powers of about $1 \mu \mathrm{W}$. Exposure to the light results in the excitation of Eosin Y to a triplet state and in turn, activates the triethanolamine co-initiator to release a neutral Eosin $\mathrm{Y}$ radical. These radicals 
initiate free-radical polymerisation reactions which convert the PEGDA pre-polymer into a hydrogel which is covalently bound to the inner surface of the HC-PCF.

\section{Experiments:}

\subsection{Hydrogel filled HC-PCF}

Following the photo-initiation process, the ends of filled HC-PCFs were analysed with assistance of a standard optical microscope, as per figure 4 (a) and (b) shown as examples of the fibres HC-PCF 1060 and HC-PCF 1550 with hydrogel. Although it is known that hydrogels can be porous [15,22] it is difficult to predict its uniformity within the HC-PCF, even if the same rule-of-thumb applies. Figure 4c, for example, shows a scanning electron microscope (SEM) image of the HC-PCF 1550 fibre with hydrogel partly formed in the core. We would anticipate that the impact of the porosity would affect the light guidance in the HCPCF, which is discussed in section 3.1.2.

(a)

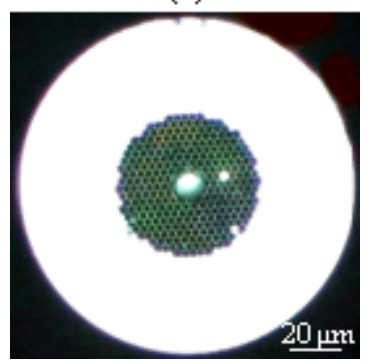

(b)

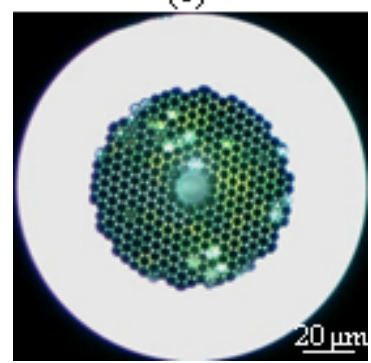

(c)

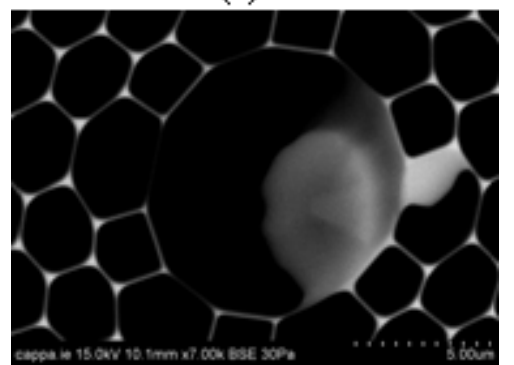

Figure 4: Cross section of fibres filled with hydrogel (a) HC-PCF 1060 (b) HC-PCF 1550 (c) SEM image of hydrogel inside the core of fibre HC-PCF 1550.

3.1.1 Spectral and near-field analyses. In order to analyse the transmission spectrum of the filled HC-PCFs after the hydrogel formation, the $530 \mathrm{~nm}$ bandpass filter was removed from the set-up and the entire supercontinuum was launched through the fibre via a $\mathrm{x} 4$ lens and its output was coupled into a multimode (MM) optical fibre for spectral analysis. For comparison, the supercontinuum spectrum (black line - Figure 5a) was obtained in the absence of the HC-PCF, but using the same lens configuration, albeit with a lateral shift of the output lens assembly. The spectral results for a $20 \mathrm{~cm}$ HC-PCF 1060 before and after filling with hydrogel are also shown in figure 5a. In this case the overall signal power was maximised. Strong attenuation was observed at all wavelengths for the empty fibre (red filled circles). In the presence of hydrogel (blue open squares), however, there was a substantial improvement in transmission for all wavelengths, especially in the infrared region (figure 5(c)), probably due to the change in refractive index. Similar effects were recently observed for aerogel filled HC-PCFs [23], where a shift in the bandgap was observed accompanied by the appearance of region of broadband guidance.

Such results are intriguing, especially for the empty fibre, as one would expect propagation for the allowed bandgap only. Therefore the setup in figure 3 was modified in order to analyse the near-field images after light propagation through the fibre samples. This was achieved using a CCD camera (LTC 0335 Series Dinion Monochrome), positioned as figure 3b. In this case, it was necessary to reduce light intensity using neutral intensity filters. It is clear from figure $5 b$ that light guidance occurred not only through the core, but also through the cladding microstructure and the solid cladding even for the empty fibre. 
(b)

(a)

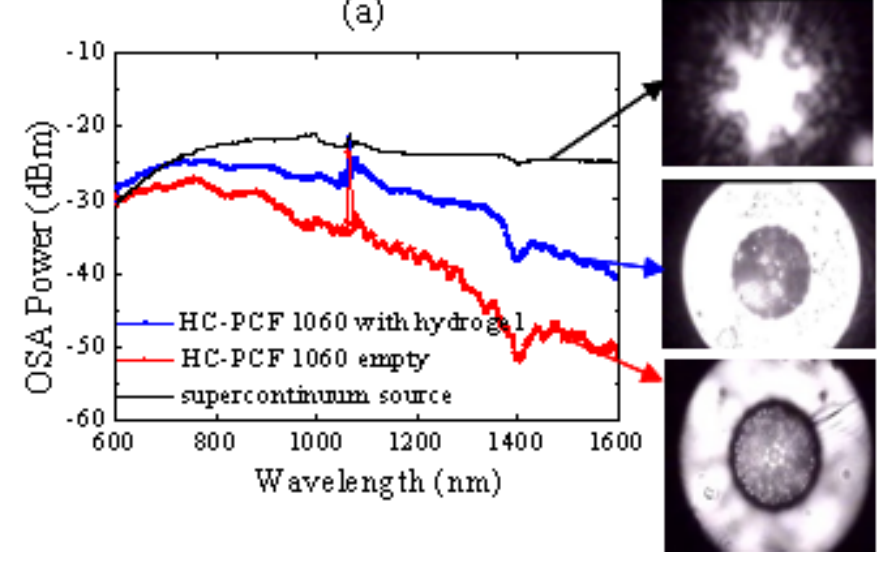

(c)

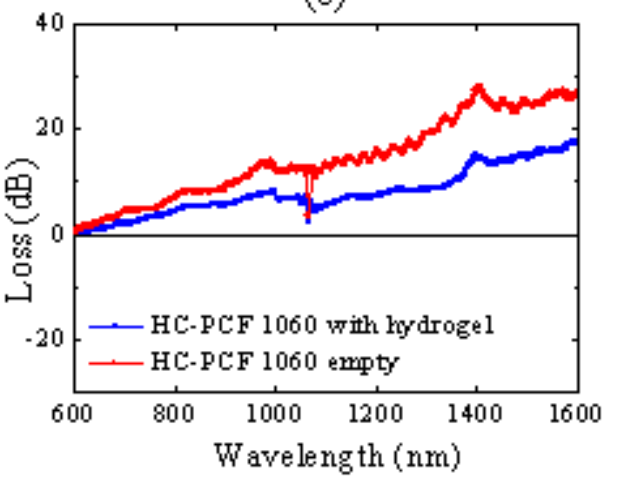

Figure 5: (a) Spectra for supercontinuum source without any fibre (black line), after empty fibre (red) and after hydrogel filled fibre (blue). (b) CCD images before HC-PCF (top), after hydrogel filled (middle) and empty (bottom) fibres. (c) Inferred insertion loss for empty (red) and hydrogel filled (blue) fibres.

In order to investigate the propagation of the broadband supercontinuum source through the filled hydrogel fibre, wavelength dependent images were taken by adding filters just before light was launched through the fibre samples. The same setup as described in section 2.3 was used, including the $\mathrm{x} 4$ focusinglens. Figure 6 below illustrates the preliminary results obtained with hydrogel filled fibre HC-PCF 1060 of approximately $46 \mathrm{~mm}$ in length. In figure 6(a), an un-filtered supercontinuum signal was transmitted, which shows that both the core and the claddin80g were inevitably excited. Filtering of the source signal, using standard bandpass filters (full width at half maximum $\sim 10 \mathrm{~nm}$ ), with the centre wavelengths at 530, 650, 800 and $1050 \mathrm{~nm}$ respectively, enabled the analysis of the transmission of specific wavelengths. In figure 6(e), where the centre wavelength is close to original bandgap $(1060 \mathrm{~nm})$ marginal light confinement was observed with no light confined in the core. At $650 \mathrm{~nm}$ and below, however, light was observed in the core of the fibre, but with substantial propagation within the cladding area due to the over illumination of the fibre outside the core. Note that at $650 \mathrm{~nm}$ the light intensity within the core was maximised, confirming the capture and guidance of light at this wavelength, and suggesting the bandgap might have shifted to this value. This guidance wavelength corresponds closely to the absorption and emissions wavelengths of the common fluorophores suitable for use of detection of DNA in a hydrogel matrix.

(a)

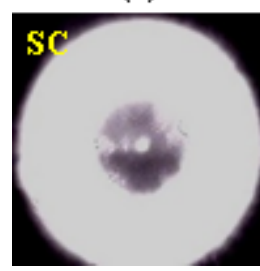

(b)

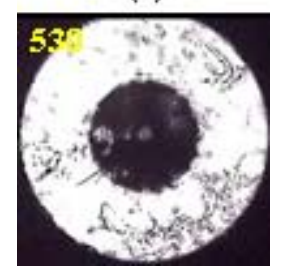

(c)

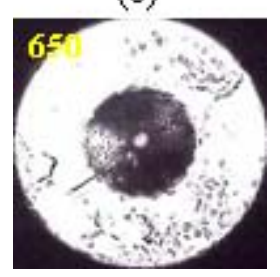

(d)

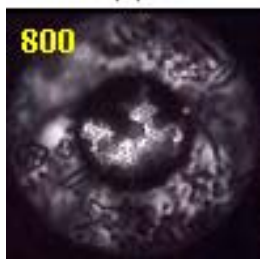

(e)

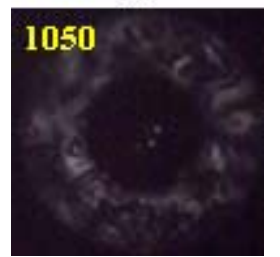

Figure 6: CCD images of an HC-PCF 1060 filled with hydrogel after transmission of a broadband supercontinuum source un-filtered (a) and filtered (b, c, d, and e). Captions indicate the centre wavelengths.

We believe that, by replacing the focusing $\mathrm{x} 4$ lens with a $\mathrm{x} 40$ lens for the optical setup shown in figure 3, it would be possible to reduce the solid cladding propagation and ensure that primarily the fibre core of a filled hydrogel HC-PCF would be illuminated. This improvement will be shown in section 3.1.2.

3.1.2 Refractive index characterisation. It is possible to estimate if there is a bandgap shift of the HC-PCF used, if the refractive index of the filling material is known [8]. In order to determine the wavelength dependence of mode propagation within the hydrogel filled fibre, the refractive index of hydrogel was estimated. For an initial estimate, hydrogel spots (diameter $7 \mathrm{~mm}$ ) were formed on a microscope slide, which 
was first modified as described in the protocol in section 2.1. Pre-polymer solution was deposited onto the silanised glass surface, and irradiated with light from an epi-fluorescence microscope filtered with a dichroic Cy3-filter (wavelength range 550/570 nm). The photo-polymerisation was initiated upon light exposure of the pre-polymer solution, using illumination times in the range from 5 to 120 seconds. These hydrogel spots were analysed using conventional ellipsometer, where changes in light polarisation can be used to determine certain properties of the material, including optical constants [24]. In this case a M-2000 Spectroscopic Ellipsometer from J.A.Woolen Co. Inc. with CompleteEASE data analysis software was used. The measurements (figure 7(a)) were significantly degraded, which we believe is due to the rough, porous surface of the hydrogel, which could have presented multiple reflecting surfaces, affecting the observed overall polarisation rotation. The estimation for $n$ and $k$ values (figure 7(b)), assuming the fitting Cauchy model from figure 7(a), and it gives a refractive index of approximately 1.46 at $600 \mathrm{~nm}$.

(a)

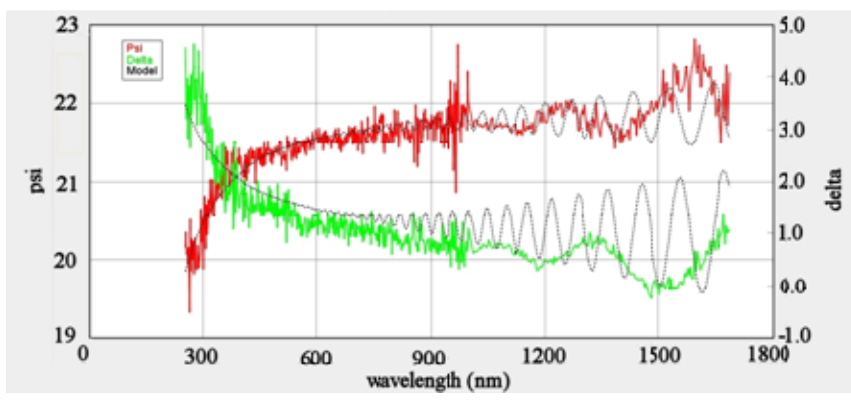

(b)

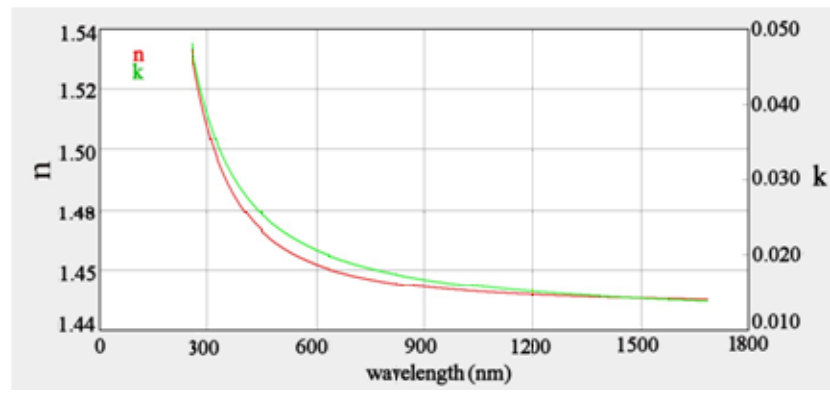

Figure 7: (a) spectroscopic ellipsometric (SE) data measurements, (b) the fitting Cauchy model for $\mathrm{n}$ and $\mathrm{k}$.

Furthermore, as seen above, the natural porosity of the hydrogel gives rise to noise in the ellipsometric results, which, in turn, could give rise to a non-uniform hydrogel distribution along the fibre length (figure 4c). The non-uniformity may be enhanced by the end illumination technique used here, due to the interaction between the partly formed hydrogel and the $530 \mathrm{~nm}$ photo-initiation signal. This may have two significant effects. Firstly, the non-uniformity will result in a significant increase in scattering, increasing the background loss of the system. Secondly, the non-uniformity will result in large variations in the bandgap shift. However, we could anticipate that the diffraction length of a signal outside the bandgap is significantly lower than the porous size, which will result in a spatially averaged bandgap shift, with a degree of residual broadening. We believe that techniques to minimise porosity [22] and improve volumetric uniformity throughout the core and microstructure cladding should minimise scattering and cladding propagation and a well-defined bandgap shift should be observed.

(a)

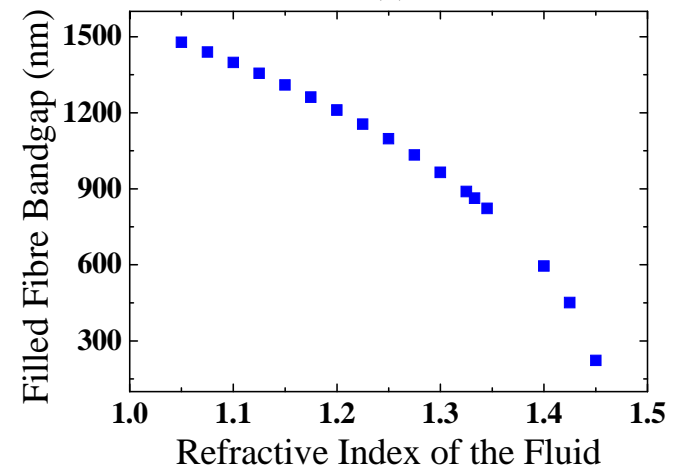

(b)

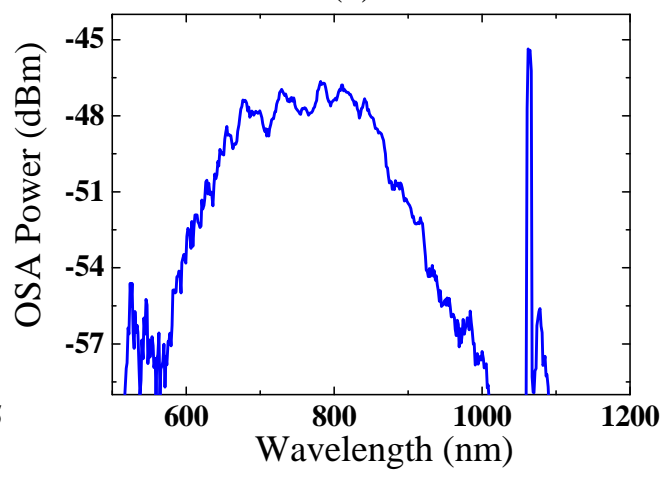

Figure 8:(a) dependence of bandgap shift of a HC-PCF 1550 on refractive index; (b) transmission spectrum of a $21 \mathrm{~cm}$ HC-PCF 1550 filled with hydrogel. 
Assuming the analysis presented in [8] which is represented in figure 8a, it seems that for the spectral dependence of the refractive index shown in figure $7 \mathrm{~b}(\sim 1.46)$, the bandgap would shift to wavelength below $200 \mathrm{~nm}$ for an HC-PCF 1060 and HC-PCF 1550, contrary to the observed wavelength shift in figure 8b. Note that, for this spectrum, a x40 launching-lens was used in order to confine the light at the filled core, unlike figure 4a. This result reveals a bandgap shift to around 700-800 nm, which would indicate a refractive index of about 1.33 . The composition of the $25 \%$ pre-polymer solution contains $75 \%$ water which gives an estimated refractive index for the hydrogel of 1.33 , which is close to that of water, for wavelength $\sim 863 \mathrm{~nm}$, as expected [8].

\subsection{DNA probe immobilisation}

The hydrogel is a 3D matrix specifically designed for detection of DNA microarrays. In this paper we report the detection of Cy5-labelled probe DNA strands immobilised with the hydrogel matrix within HC-PCFs. In order to immobilise probe DNA within the hydrogel, amino-modified oligonucleotides (Metabion International AG) were added to a volume of the pre-polymer solution to give a concentration of $260 \mathrm{nM}$. Roughly $0.1 \mathrm{mg}$ of NHS-PEG-acrylate cross-linker in powdered form (Nektar Therapeutics, Huntsville, AL) was added to this mixture, in order to provide an anchor or binding site for the probe sequence. The formation of the hydrogel and the immobilisation of probe DNA occur simultaneously during the photo-polymerisation process (as per section 2.3), as the NHS-PEG-acrylate cross-linker allows for the covalent attachment of the probe to the polymer backbone.

(b)

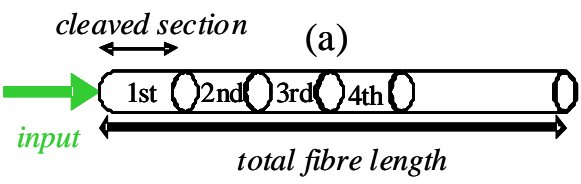

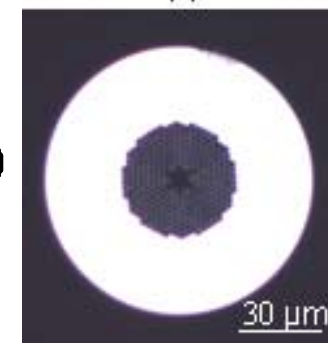

(c)

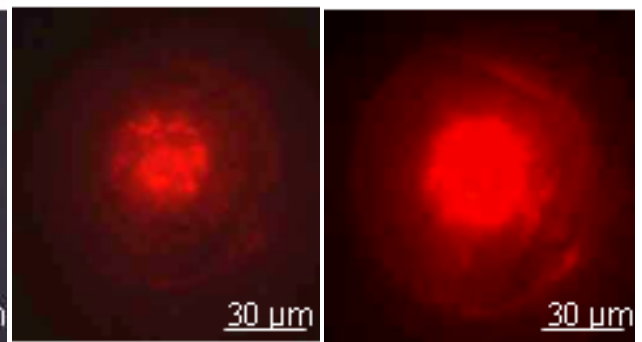

Figure 9: (a) Fibre cleaving schematic. Also showing cross-section images (x20 magnification) of a HC-PCF 1060 for (b) an empty fibre as a visual guide; and Cy5-fluorescence for hydrogel and DNA filled fibres with

(c) $20 \mathrm{~ms}$ and (d) $50 \mathrm{~ms}$ integration times.

A standard fluorescence microscope (Olympus BX51 with Cell^₹ software) was used to analyse the fluorescence effectively and reproducibly. The microscope contained the appropriate dichroic filters to excite the Cy5-fluorophore $(\sim 650 \mathrm{~nm})$ and detect only the fluorescence $(\sim 670 \mathrm{~nm})$. However, it was not possible to analyse the entire length of fibre, and therefore, for this preliminary experiment, short pieces of the prepared fibres $(0.3$ to $1 \mathrm{~cm}$ ) were cleaved (figure $9(\mathrm{a}))$ and mounted vertically on a microscope slide. Precise cleaving and dust-free surfaces were paramount. Figure 9 (c) and (d) shows the cross-section of the $4^{\text {th }}$ centimetre cut from input of a HC-PCF 1060 and the Cy5 fluorescence analysis for two different integration times.

(a)

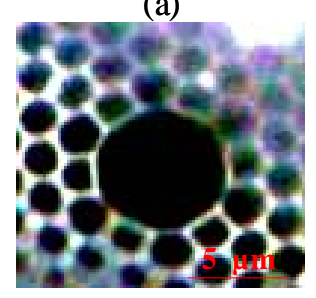

Figure 10: Cross-section images (x100 magnification) of a HC-PCF 1550 for (a) an empty fibre, and (b) for hydrogel and Cy5 labelled DNA filled fibre with 100 ms integration time. (b)

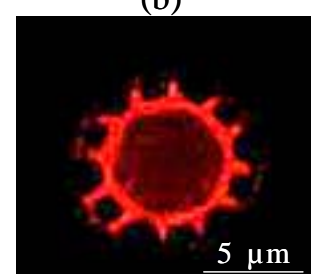


The same analysis was performed with a HC-PCF 1550 sample. Figure 10 shows the results obtained with a $0.3 \mathrm{~cm}$ length sample, x100 magnification and $100 \mathrm{~ms}$ integration time. Although the fibre sample was extremely short, it was still possible to detect the Cy5-fluorescence. Note that, in the case of the HC-PCF $1550 \mathrm{~nm}$ fibre, the shifted bandgap did not correspond to the fluorescence wavelength, and the detected fluorescence appears to be guided by the solid elements of the microstructure. However, it is also possible that the launch conditions (x100 lens) lead to increased coupling to the cladding modes. Re-optimisation of the launch, taking into account any changes in fibre N.A. due to the presence of the hydrogel, may improve these results.

It is important, however, to distinguish between the results obtained above and auto-fluorescence, as hydrogels are known to be highly fluorescent in the visible range, due to the use of Eosin $\mathrm{Y}$ as a photoinitiator for the silanisation process [16]. The hydrogel auto-fluorescence was detected for Cy3 dichroic filters, instead of Cy5, where the excitation wavelength is $\sim 550 \mathrm{~nm}$ and the emission at $\sim 570 \mathrm{~nm}$. The hydrogel filled fibre with and without labelled DNA microarrays inside its structure were analysed. Figure 11 shows an example of the detected auto-fluorescence of the hydrogel in the fibre in comparison to Cy-5 labelled DNA-probe immobilisation detection. Hydrogel auto-fluorescence is observed in figure 11b at the locations suggested by the optical image of the fibre (figure 11(a)). Although fluorescence is only observed at locations corresponding to hydrogel auto-fluorescence, not all hydrogel sites exhibited Cy-5 fluorescence, consistent with the randomness of the DNA immobilisation.

(a)

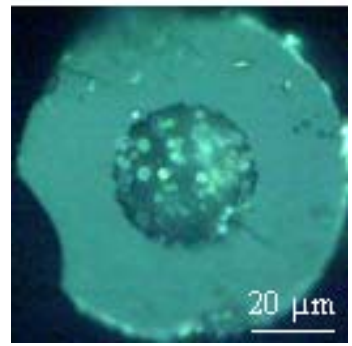

(b)

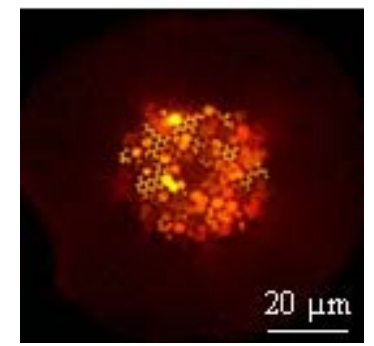

(c)

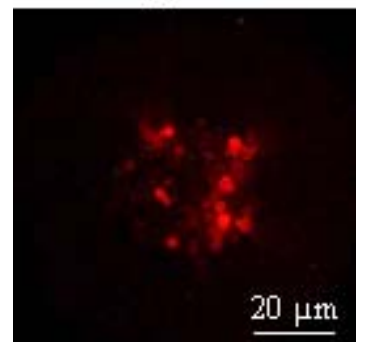

Figure 11: Cross section images (x100) magnification of a HC-PCF 1060, for (a) bright light, (b) for Cy3 dichroic filter, with integration time 2ms (c) for Cy5 dichroic filter, with the integration time $2 \mathrm{~s}$.

In figure 12 the hydrogel is formed within the core and cladding holes containing no probe DNA molecules. The hydrogel auto-fluorescence was observed in the range $\sim 570 \mathrm{~nm}$ as expected, while there was no light detection in the range $\sim 670 \mathrm{~nm}$ due to absence of Cy5 tagged DNA.
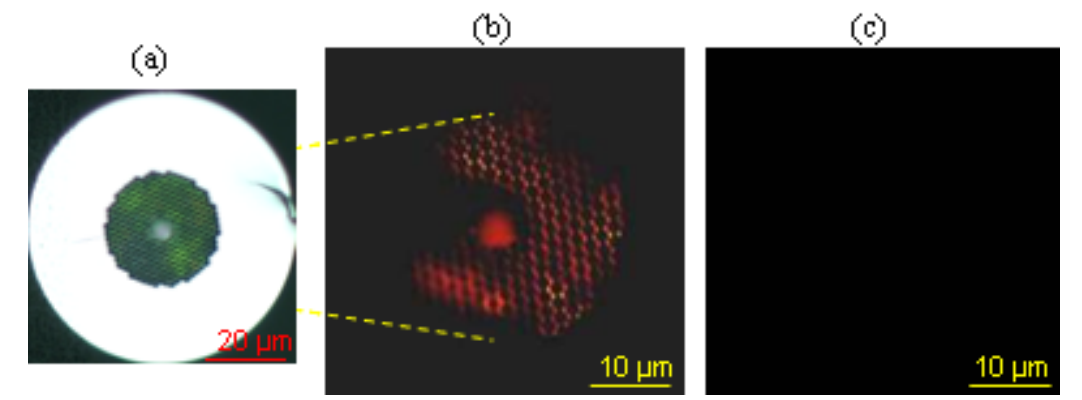

Figure 12: Cross section images of a HC-PCF 1060, for (a) bright light, with (x50) magnification, and (b) (x100) magnification for Cy3 dichroic filter, with integration time 2ms (c) (x100) magnification for Cy5 dichroic filter, with the integration time $2 \mathrm{~s}$.

\section{Conclusion}

We have demonstrated the growth of a 3D hydrogel matrix within the holes of a Hollow-Core Photonic Crystal Fibre. The light behaviour of these hydrogel filled fibres and the estimated refractive index of the hydrogel is in good agreement with theoretical discussions. Moreover Cy5-labelled DNA immobilisation and 
hydrogel auto-fluorescence inside HC-PCF structure were detected. The integration of the hydrogel with PCF is a promising technique for highly sensitive bio-detection devices. Such a scheme would provide a compact solution as a point-of-care device for rapid detection of bio samples.

\section{Acknowledgements}

The authors would like to acknowledge Rod Webb, Vladimir Djara, Jonathan Ward and Eileen Hurley from Tyndall National Institute, and Kieran Deasy, Liam Lewis, and Eamon O'Neill from CIT, for support and useful discussion. This material is based upon work supported by the Science Foundation Ireland under grant number 06/IN/I969 and Enterprise Ireland grant numbers TD/03/123 and PC/2008/30. The authors also wish to acknowledge support from the Irish Higher Education Authority's Programme for Research in Third Level Institutions (PRTLI) which facilitated this research.

\section{References}

[1] Russell P 2003 Photonic crystal fibres Science 299 358-362

[2] Monro T M, Belardi W, Furusawa K, Baggett J C, Broderick N G R and Richardson D J 2001 Sensing with microstructured optical fibres Meas. Sci. Technol. 12 854-858

[3] Broeng J, Barkou S E, Søndergaard T, and Bjarklev A 2000 Analysis of air-guiding photonic bandgap fibers Opt. Lett. 25 96-98

[4] Benabid F 2006 Hollow-core photonic bandgap fibre: new light guidance for new science and technology Phil.Trans. R. Soc 364 3439-62

[5] Benabid F and Roberts P J 2008 Guidance mechanisms in hollow-core photonic crystal fiber Proc. SPIE. 69010U $1-10$

[6] Knight J C 2003 Photonic crystal fibres Nature, 424, 847-851

[7] Bouwmans G, Luan F, Knight J C, Russell P St J 2003 Properties of a hollow-core photonic bandgap fibre at $850 \mathrm{~nm}$ wavelength Opt. Exp. 11, 1613-20

[8] Antonopoulos G, Benabid F, Birks T A, Bird D M, Knight J C and Russell P St J 2006 Experimental demonstration of the frequency shift of bandgaps in photonic crystal fibers due to refractive index scaling Opt. Exp. 14, 3000-06

[9] Jensen J B, Pedersen L H, Hoiby P E, Nielsen L B, Hansen T P, Folkenberg J R, Riishede J, Noordegraaf D, Nielsen K, Carlsen A, Bjarklev A 2004 Photonic crystal fiber based evanescent-wave sensor for detection of biomolecules in aqueous solutions Opt. Lett. 29 1974-76

[10] Shahraam A V, Warren-Smith S C, Monro T M 2007 Enhancement of fluorescence-based sensing using microstructured optical fibres Opt. Exp. 15 17891-901

[11]Jensen J B, Hoiby P E, Emiliyanov G, Bang O, Pedersen L H, Bjarklev A 2005 Selective detection of antibodies in microstructured polymer optical fibres Opt. Exp. 13 5883-89

[12] Ruan Y, Foo T Ch, Warren-Smith S, Hoffmann P, Moore R C, Ebendorff-Heidepriem H, Monro T M 2008 Antibody immobilization within glass microstructured fibres: a route to sensitive and selective biosensors Opt. Exp. 16 18514-523

[13] Myaing M T, Ye J Y and Norris T B, Thomas T, Baker J R, Jr, Wadsworth W J, Bouwmans G, Knight J C, Russell P St J 2003 Enhanced two-photon biosensing with double-clad photonic crystal fibres Opt. Lett. 18 1224-26

[14] Hoiby P E, Nielsen L B, Jensen J B, Hansen T P, Bjarklev A, Pedersen L H 2004 Molecular immobilization and detection in a photonic crystal fiber Proc. SPIE 5317 220-223.

[15] Dyukova V I, Dementieva E I, Zubtsov D A, Galanina O E, Bovin N V, Rubina A Yu 2005 Hydrogel glycan microarrays Anal. Biochem 347 94-105

[16] Charles P T, Goldman E R, Rangasammy J G, Schauer C L, Chen M-S, Taitt C R 2004 Fabrication and characterization of 3D hydrogel microarrays to measure antigenicity and antibody functionality for biosensor applications Biosens. And Bioelect 20 753-764 
[17] Yershov G, Barsky V, Belgovskiy A, Kirllov E, Kreindlin E, Ivanov I, parinov S, Guschin D, Drobishev A, Dubiley S, Mirzabekov A 1996 DNA analysis and diagnostic on oligonucleotides microchips” Proc.Natl. Acad. Sci. USA, 93, 4913-4918

[18] Kizilel S, Pérez-Luna V H, Teymour F 2004 Photopolymerization of Poly(Ethylene Glycol) Diacrylate on Eosin-Functionalized Surfaces Langmuir 20 8652-58

[19] Rutowska M, Horan L, Gunning F C G 2009 Photonic Crystal Fibres: Selective Filling Delays CLEOEurope CH.P.20THU

[20] Moore E, O’Connell D, Galvin P 2006 Surface characterisation of indium-tin oxide thin electrode films for use as a conducting substrate in DNA sensor development Thin Sol. Fil. 515 2612-17

[21] Dudley J M, Provino L, Grossard N, Maillotte H 2002 Supercontinuum generation in air-silica microstructured fibres with nanosecond and femtosecond pulse pumping J. Opt. Soc. Am. B 19 765-771

[22] Ehrick J D, Deo S K, Browning T W, Bachas L G, Madou M J, Daunert S 2005 Genetically engineered protein in hydrogels tailors stimuli-responsive characteristics Nature 4 298-302

[23] Grogan M D W, Leon-Saval S G, Williams R, England R, Birks T A 2009 Optical Fibre with an Aerogel-Filled Core Proc. CLEO/IQEC CMW3

[24]Woollam J A, Johns B D, Herzinger C M, Hilfiker J M, Synowicki R A, Bungay C L 1999 Overview of variable angle spectroscopic ellipsometry (VASE), Part I: Basic Theory and Typical Applications”. Proc.

SPIE .CR72 3-28 